\section{Social cognition in schizophrenia}

\author{
Thammanard Charernboon, 1,2 \\ Jayanton Patumanond 2 \\ 1Department of Psychiatry, and \\ 2Division of Clinical Epidemiology, \\ Faculty of Medicine, Thammasat \\ University, Pathumthani, Thailand
}

\begin{abstract}
Impairments in social cognitions in schizophrenia are increasingly reported in the last decade but only a few studies have come from Asia. The objective of the study was to evaluated emotion perception, theory of mind and social knowledge in people with schizophrenia compared to healthy controls. Participants were 36 clinically stable outpatients with schizophrenia and 36 normal controls with comparable age and level of education. We administered general neurocognition test (the Addenbrooke's Cognitive Examination), emotion perception (the Faces Test), theory of mind (the Eyes Test) and social knowledge (the Situational Features Recognition Test; SFRT). Schizophrenia patients displayed obvious impairment in all three social cognition domains i.e. the Faces Test [13.7 (2.9) vs 15.7 (1.9), $\mathrm{P}=0.001]$, the Eyes Test [18.9 (4.4) vs 23.5 (4.4), $\mathrm{P}<0.001]$ and SFRT [0.85 (0.09) vs 0.9 (0.05), $\mathrm{P}=0.002]$. The performances on three social cognition tests did not correlate with positive symptoms. Only the Faces Test seemed to be related to negative symptoms. The results demonstrated that there are deficits of social cognitions in schizophrenia even in a clinically stable population.
\end{abstract}

\section{Introduction}

In the past three decades, studies in schizophrenia have focused on neurocognitive function. The results consistently demonstrated that neurocognitive deficits are found in almost all schizophrenia patients and are a core feature of the disorder.1,2 However, in the last decade, cognitive neuroscience studies in schizophrenia shifted attention from neurocognitive function towards social cognition, a relatively new concept, which seems to be a better predictor of social abilities and real world function.3,4 Social cognition refers to the mental operations underlying social interaction. These processes generally consist of understanding other people's emotion, mental states, intentions, and actions in social settings. ${ }^{5}$ The
Measurement and Treatment Research to Improve Cognition in Schizophrenia (MATRICS) and Cognitive Neuroscience for Treatment Research to Improve Cognition in Schizophrenia (CNTRICS) have specified five areas of social cognition for schizophrenia as emotion perception, theory of mind, social knowledge, social perception and attributional biases. 6,7 From the recent meta-analysis studies, theory of mind and emotion perception are the most extensively studied domains in people with schizophrenia compared to normal controls. ${ }^{4,8}$ Emotion perception refers to the ability to identify the emotions of others, and theory of mind refers to the ability to understand other people's mental states and feeling. The meta-analysis showed that schizophrenia patients performed significantly worse than healthy individuals with large effect sizes for emotion perception (Hedge's: $g=0.89$ ) and theory of mind ( $g=0.96) .{ }^{8}$ However, most previous studies in emotion perception and theory of mind have been from the West, and the results suggest that there might be an impact of race and culture on social cognition. ${ }^{9}$ The reviews demonstrated that studies in Caucasians are overrepresented versus other races, and only a few studies have come from Asia. 8,9 On the other hand, it should be noted that social knowledge, an awareness of social situation, rules, roles, goals, and expectation, is less studied as compared to the two prior domains. Salva's meta-analysis included only seven studies and found that people with schizophrenia had impairment in social knowledge with a medium effect size $(\mathrm{g}=0.54) .{ }^{8}$ The current study has two objectives. The primary objective was to evaluate emotion perception, theory of mind and social knowledge in clinically-stable schizophrenia patients compared to healthy controls. We hypothesized that schizophrenia patients would have lower scores in social cognition tests than the normal controls. The secondary objective was to explore the relationship between residual positive symptoms, negative symptoms, and neurocognition with social cognitions.

\section{Materials and Methods}

\section{Participants}

There were 72 participants which consisted of 36 people with schizophrenia and 36 healthy controls. They were recruited on a voluntary basis with consecutive sampling method. All participants were
Correspondence: Thammanard Charernboon, Department of Psychiatry, Faculty of Medicine, Phahon Yothin Road, Thammasat University, Pathumthani 12120, Thailand. Tel.: +66.2.926.9204

E-mail: dr.thammanard@gmail.com

Key words: Emotion perception; schizophrenia; social cognition; social knowledge; theory of mind.

Acknowledgements: the authors would thank Tiraya Lerthattasilp, MD and Piyanud Chompookard, BA, for help in collecting the data.

Contributions: TC, concept and design of the study, collection of data, statistical analysis, writing the manuscript, final approval of the article; JP, study design, statistical analysis, final approval of the article.

Conflict of interest: the authors declare no potential conflict of interest.

Received for publication: 23 January 2017.

Revision received: 5 March 2017.

Accepted for publication: 6 March 2017

This work is licensed under a Creative Commons Attribution-NonCommercial 4.0 International License (CC BY-NC 4.0).

CC Copyright T. Charernboon and J. Patumanond, 2017 Licensee PAGEPress, Italy

Mental Illness 2017; 9:7054

doi:10.4081/mi.2017.7054

Thai literate, age between 20-60 year-old and had at least 6 years of formal education. Schizophrenia patients and normal controls participants were matched 1:1 using comparable age and educational level.

\section{Schizophrenia patients}

Patients were recruited from the Outpatient Psychiatry Clinic, Thammasat University Hospital, Thailand during 1 May 2016 to 31 December 2016. They were diagnosed with schizophrenia according to the Diagnostic and Statistical Manual of Mental Disorders, Fifth Edition criteria (DSM-5) by the consensus of two psychiatrists; one research psychiatrist (TC) and other psychiatrists who were in charge of participating cases reviewed medical records and assessed the participants on the same day. All the participants were clinically stable as defined by no significant change in the symptoms and treatment in the last three months. A history of substance dependencies (except for nicotine) and major neurological disorders were exclusion criteria. 


\section{Normal controls}

Healthy controls were recruited from patients or relative of the patients from other clinics in Thammasat University Hospital. They had no history of major psychiatric disorders (i.e. schizoprenia, bipolar disorder, major depressive disorder and substance disorders) nor major neurological disorders.

\section{Ethics}

All participants gave written consent. The study was approved by the Human Ethics Committee of Thammasat University (protocol number: MTU-EC-ES-6-043/59).

\section{Measures}

\section{Emotion perception: the Faces Test}

The Faces Test is composed of 20 full facial photographs in a variety of emotional expressions. ${ }^{10}$ Participants had to select a correct answer from multiple choices. The maximum total score is 20. Higher scores indicate better emotion perception ability.

\section{Theory of mind: the Reading the Mind in the Eyes (the Eyes Test)}

The Eyes Test is a widely used measure of theory of mind in schizophrenia studies. It includes 36 pictures of the eye regions of various faces. The task involves choosing the words that best match to what the person in the picture is thinking or feeling. ${ }^{11}$ The maximum total score of the Eyes Test is 36 . Higher scores indicate a better theory of mind.

\section{Social knowledge: the Situational Features Recognition Test}

The SFRT required participants to identify features from a list for situations (e.g. taking a test, reading in a library and getting a haircut). The SFRT total score range from 0 to 1 . The formula to calculate the total score is fully described in a Corrigan et al. (1996). ${ }^{12}$ Higher scores indicate better social knowledge.

\section{Neurocognition: the Addenbrooke's Cognitive Examination}

The ACE is used as a measure of general neurocognitive function. It assesses five neurocognitive areas: attention and orientation, verbal fluency, language, visuospatial ability and memory. ${ }^{13,14}$ The score range from 0 to 100 , the higher score indicates better neurocognition. The cut-off score to distinguish the cognitively healthy controls from mild cognitive impairment was $75 / 76.13$

\section{Positive and negative symptoms}

The Scale for the Assessment of Positive Symptoms (SAPS) and the Scale for the Assessment of Negative Symptoms (SANS) were administered to the patients to assess symptom severity. 15 SAPS had a global rating score of between 0-20 and SANS had a global rating score of between $0-25$. Higher scores indicate more severe symptoms.

\section{Procedure}

Demographic and clinical data were collected. Clinical symptoms in the schizophrenia group were rated prior to the neurocognition and social cognition tests using the SAPS and SANS by a research psychiatrist (TC) for all patients. Then the ACE and social cognition tests were administered by an independent psychologist (PC) or psychiatrist (TL).

\section{Data analysis}

Participants' characteristics were tested with exact test and t-test. Performances in social cognition tests between groups were analyzed with t-test. Effect size of each social cognition tests were also calculated using Cohen's d scores. Multivariable regression analysis was carried out to correct the effect of characteristic factors. Intercorrelation between clinical measures, neurocognition and social cognition in the schizophrenia patients and healthy participants were analyzed using Pearson's correlation. The P-value was set to 0.05 .

\section{Sample size}

Based on testing two independent means formula, a minimum sample size of 36 participants per group would be need to achieve $90 \%$ power to detect the difference of the Eyes Test if the true difference between the patients and controls is 3.0, with the standard deviation of 3.8.16

\section{Results}

Table 1 summarized participants' characteristics and clinical symptoms. Most of the participants were female $(66.7 \%)$. People with schizophrenia showed no statistic differences from the normal controls in gender, age and level of education. The mean global rating SAPS score of the patients was 2.8(2.4) and SANS score was 7.5 (5.1). Thirteen patients (36.1\%) had no positive symptoms (as indicated by SAPS score $\leq 1$ in every symptom), and only one patient $(2.8 \%)$ had no negative symptoms.

Table 1. Sample characteristics.

\begin{tabular}{lccc} 
Variable & $\begin{array}{c}\text { Schivophrenia } \\
(\mathrm{n}=36)\end{array}$ & $\begin{array}{c}\text { Normal controls } \\
(\mathrm{n}=36)\end{array}$ & $\begin{array}{c}\text { P-value } \\
\text { Gender: female, N (\%) }\end{array}$ \\
$\begin{array}{l}22(55.6) \\
\text { Age (years), mean (SD) }\end{array}$ & $37.1(11.2)$ & $37.1(12.1)$ & 0.22 \\
\hline Education (years), mean (SD) & $13.8(3.7)$ & $13.8(4.2)$ & 0.984 \\
\hline Duration (years), mean (SD) & $8.2(8.1)$ & - & 0.976 \\
\hline Number of psychotic episodes, mean (SD) & $2.1(1.0)$ & - & - \\
Positive symptoms (SAPS), mean SD & $2.8(2.4)$ & - & - \\
\hline Negative symptoms (SANS), mean SD & $7.5(5.1)$ & - & - \\
Typical antipsychotic N (\%) & $27(75)$ & - & - \\
\hline Atypical antipsychotic N (\%) & $12(33.3)$ & - \\
Anticholinergic drugs N (\%) & $10(27.8)$ & - \\
\hline
\end{tabular}

SD, standard deviation; SAPS, Scale for the Assessment of Positive Symptoms; SANS, Scale for the Assessment of Negative Symptoms.

Table 2. Performance in neurocognition and social cognition tests.

\begin{tabular}{lcccc} 
& Schirophrenia, mean (SD) & Normal controls, mean (SD) & Effect size (95\%CI) & P-value \\
ACE: mean (SD) & $82.3(10.7)$ & $92.1(6.7)$ & $1.11(0.61-1.6)$ & $<0.001$ \\
The Faces Test: mean (SD) & $13.7(2.9)$ & $15.7(1.9)$ & $0.81(0.32-1.29)$ & 0.001 \\
\hline The Eyes Test: mean (SD) & $18.9(4.4)$ & $23.5(4.4)$ & $1.05(0.55-1.54)$ & $<0.001$ \\
SFRT: mean (SD) & $0.85(0.09)$ & $0.90(0.05)$ & $0.77(0.29-1.24)$ & 0.002 \\
\hline
\end{tabular}

ACE, the Addenbrooke's Cognitive Examination; SFRT, the Situational Features Recognition Test. 
All patients received antipsychotics: 24 received typical antipsychotics, and 9 received atypical antipsychotics. Three patients received combined typical and atypical antipsychotics. Only 10 patients received anticholinergic medications. Regarding neurocognition, schizophrenia patients attained significantly lower ACE scores than normal controls; however, they were still within normal range.

The patients' group demonstrated significant deficits relative to the healthy control group across all three measures of social cognition (Table 2). The effect sizes for the differences of emotion perception and theory of mind were large, but medium in social knowledge. After controlling for the influence of gender, age and level of education, multivariable regression analysis confirmed that deficits in social cognition remained significant (Table 3). Education level was another significant predictor of performance in all three social cognition tests, whereas gender did not predict performance in these tests, and age was marginally predictive of performance only in The Faces Test. Regarding the correlation between social cognition tests, the Faces Test, the Eyes Test and SFRT correlated with each other in both healthy individuals and schizophrenia patients with the correlation coefficient between 0.41-0.69 (Table 4). All social cognition tests were found to be correlated with the ACE in both groups. SFRT seem to have the highest correlation with the ACE with a correlation coefficient of 0.62 and 0.67 , while the lowest was the Eyes Test with a correlation coefficient of 0.49 and 0.58 . Positive symptoms did not correlate with any of the social cognition tests. Negative symptoms significantly correlated with the Faces Test, but not the Eyes Test and SFRT.

\section{Discussion and Conclusions}

The advantage of this study was the matching for age and level of education between normal controls and patients, and inclusion of three measures that target different aspects of social cognitions.

Schizophrenia patients displayed obvious impairment in all three social cognition domains i.e. emotion perception, theory of mind and social knowledge, and they are not explained by the influence of potential predictors such as age, gender and educational level. The results of our study, which included clinically stable schizophrenia with low positive symptoms scores, suggest that social cognition impairment possibly represents one of the core symptoms and a trait characteristic of schizophrenia. The results are in agreement with most studies that schizophrenia is characterized by a deficit in social cognition..$^{8,17-19}$
The effect size for theory of mind and emotion perception in our study was large (1.05 and 0.81 , respectively) which was comparable to the previous meta-analysis showing effect sizes of 0.96 and 0.89 , respectively. ${ }^{8}$ Another meta-analysis of theory of mind in schizophrenia by Bora et al. which reported the sensitivity analysis of only the Eyes Test from eight studies also had an effect size of 0.9. ${ }^{19}$ For social knowledge, the effect size was found to be lower than the other two domains which was in concordance with the previous study. The present study demonstrated a slightly higher effect size than in the meta-analysis. (0.77 vs 0.54$).{ }^{8}$ Differences in social knowledge measures used might explain this small divergence in findings.

Comparing to other similar studies in Asia, for example, a study by Hirao et al., Japan, using the Eyes Test revealed that people with schizophrenia performed worse

Table 3. Multivariable regression analysis of factors predicting performance in social cognition tests.

\begin{tabular}{lcc}
\hline Variable & Coefficients & P-value \\
The Faces Test & & $<0.001$ \\
Schizophrenia & -1.99 & 0.966 \\
Gender: female & 0.02 & 0.039 \\
Age & -0.05 & 0.004 \\
Education & 0.22 & \\
The Eyes Test & & $<0.001$ \\
Schizophrenia & -4.74 & 0.359 \\
Gender:female & -0.83 & 0.272 \\
Age & -0.05 & $<0.001$ \\
Education & 0.58 & \\
\hline SFRT & & $<0.001$ \\
Schizophrenia & -0.056 & 0.236 \\
Gender: female & -0.017 & 0.783 \\
Age & -0.0002 & $<0.001$ \\
Education & 0.009 &
\end{tabular}

SFRT, the Situational Features Recognition Test.

Table 4. Intercorrelation between clinical symptoms, neurocognition and social cognition tests.

\begin{tabular}{|c|c|c|c|c|c|c|}
\hline & $1 \mathrm{ACE}$ & 2 Faces Test & 3 Eyes Test & 4 SFRT & 5 SAPS & 6 SANS \\
\hline \multicolumn{7}{|c|}{ Healthy individuals } \\
\hline 1. ACE & 1.0 & & & & & \\
\hline 2. The Faces Test & $0.58^{* * *}$ & 1.0 & & & & \\
\hline 3. The Eyes Test & $0.58^{* * *}$ & 0.29 & 1.0 & & & \\
\hline 4. SFRT & $0.62^{* * *}$ & $0.41^{*}$ & $0.48^{* *}$ & 1.0 & & \\
\hline
\end{tabular}

\begin{tabular}{lccccc} 
1. ACE & 1.0 & & & & \\
2. The Faces Test & $0.52^{* *}$ & 1.0 & 1.0 & & \\
\hline 3. The Eyes Test & $0.49^{* *}$ & $0.43^{* *}$ & $0.69^{* * *}$ & 1.0 & 1.0 \\
4. SFRT & $0.67^{* * *}$ & $0.46^{* *}$ & 0.12 & 0.03 & -0.26 \\
\hline 5. SAPS & -0.08 & -0.01 & -0.04 & 0.11 & 1.0 \\
6. SANS & $-0.5^{* *}$ & $-0.45^{* *}$ &
\end{tabular}

ACE, the Addenbrooke's Cognitive Examination; SFRT, the Situational Features Recognition Test; SAPS, Scale for the Assessment of Positive Symptoms; SANS, Scale for the Assessment of Negative Symptoms. ${ }^{*} \mathrm{P}<0.05,{ }^{*} \mathrm{P}<0.01,{ }^{* * *} \mathrm{P}<0.001$ 
than healthy controls (mean accuracy $53.2 \%$ vs $67.6 \%$ ) with the mean accuracy comparable to this study $(52.5 \%$ vs $65.3 \%) .{ }^{20}$ A study of emotion perception in China by $\mathrm{Li}$ et al. which used the Facial Emotion Identification Test also revealed a comparable deficit in emotion perception for schizophrenia patients with the effect size of 0.79 .21 The finding of our study implies that impairments in social cognition represent a core deficit in schizophrenia regardless of race or culture.

As for the association between the social cognition tests, our study showed moderate correlations between emotion perception, theory of mind and social knowledge in both normal controls and patients. This might support that they are related dimensions yet not exactly the same concept. Social cognition tasks usually recruit several processes with general neurocognition and other sub-domains of social cognition. For example, by theory, correct indentation of the theory of mind in the Eyes Test may require an emotion perception ability. 2,5 All social cognition tests were correlated with general neurocognition in both healthy participants and patients. In principle, social cognition and neurocognition use overlapping cognitive process, e.g. attention/concentration and language; consequently, both types of cognitive function are often correlated in some degree. ${ }^{17,19}$

Our study showed that all social cognition did not correlate with positive symptoms. Many studies have demonstrated that social cognition impairments do not seem to be related to psychotic symptoms in remitted patients.2,8,22 For negative symptoms, only the Faces Test correlated with negative symptoms. The previous studies reported an inverse relationship between negative symptoms and theory of mind.16,23 In contrast, other researches e.g. de Achaval et al., which used the Eyes Test demonstrated no correlation. ${ }^{2}$ It remains unclear whether our findings might due to insufficient power to detect the correlation. Moreover, differences in measurement difficulty and clinical assessment tools may be alternate reasons as well.

\section{Clinical implications}

The impairments in social cognition detected may explain the deficits in social skills and social functions of people with schizophrenia. This is because they have less ability to detect others' emotion, correct appraisal of the feelings or intentions of others, and understand social rules. The study underscores the importance of assessing social cognition in people with schizophrenia.

\section{Limitations}

The main limitation of the study was the wide variety in duration of illness in the patient group, which may have accounted for the range in severity of social cognition deficits. Although only $28 \%$ of patients used anticholinergic drugs, it may have affected the performance in neurocognition and social cognition. In addition, the sample size may not be adequate to detect the correlation between negative symptoms, positive symptoms, neurocognition and social cognition.

Prospective studies over a long period in first-episode schizophrenia patients are needed to fully demonstrate the predictors of social cognition deterioration. It might also to be fruitful to investigate other social cognition domains e.g. social perception and attributional biases in Asian countries.

\section{References}

1. Insel TR. Rethinking schizophrenia. Nature 2010;468:187-93.

2. de Achával D, Costanzo EY, Villarreal M, et al. Emotion processing and theory of mind in schizophrenia patients and their unaffected first-degree relatives. Neuropsychologia 2010;48:1209-15.

3. Fett A-KJ, Viechtbauer W, Penn DL, et al. The relationship between neurocognition and social cognition with functional outcomes in schizophrenia: a meta-analysis. Neurosci Biobehav Rev 2011;35: 573-88.

4. Penn DL, Sanna LJ, Roberts DL. Social cognition in schizophrenia: an overview. Schizophr Bull 2008;34:408-11.

5. Billeke P, Aboitiz F. Social cognition in schizophrenia: from social stimuli processing to social engagement. Front Psychiatry 2013;4:4.

6. Green MF, Penn DL, Bentall R, et al. Social cognition in schizophrenia: an NIMH workshop on definitions, assessment, and research opportunities. Schizophr Bull 2008;34:1211-20.

7. Kirkpatrick B, Fenton WS, Carpenter WT, Marder SR. The NIMH-MATRICS consensus statement on negative symptoms. Schizophr Bull 2006;32:214-9.

8. Savla GN, Vella L, Armstrong CC, et al. Deficits in domains of social cognition in schizophrenia: a meta-analysis of the empirical evidence. Schizophr Bull 2012;39:979-92.

9. Irani F, Seligman S, Kamath V, et al. A meta-analysis of emotion perception and functional outcomes in schizophrenia. Schizophr Res 2012;137:203-11.

10. Baron-Cohen S, Wheelwright S, Jolliffe,
Therese. Is there a language of the eyes? Evidence from normal adults, and adults with autism or Asperger syndrome. Vis Cogn 1997;4:311-31.

11. Baron-Cohen S, Wheelwright S, Hill J, et al. The Reading the Mind in the Eyes test revised version: a study with normal adults, and adults with Asperger syndrome or high-functioning autism. J Child Psychol Psychiatry 2001;42:24151.

12. Corrigan PW, Silverman R, Stephenson $\mathrm{J}$, et al. Situational familiarity and feature recognition in schizophrenia. Schizophr Bull 1996;22:153-61.

13. Charernboon T, Jaisin K, Lerthattasilp T. The Thai version of the Addenbrooke's cognitive examination III. Psychiatry Investig 2016;13:571-3.

14. Hsieh S, Schubert S, Hoon C, et al. Validation of the Addenbrooke's cognitive examination III in frontotemporal dementia and Alzheimer's disease. Dement Geriatr Cogn Disord 2013;36: 242-50.

15. Andreasen NC. Scale for the Assessment of Negative Symptoms (SANS). Br J Psychiatry 1989;7:49-58.

16. Bora E, Gökçen S, Kayahan B, Veznedaroglu B. Deficits of social-cognitive and social-perceptual aspects of theory of mind in remitted patients with schizophrenia: effect of residual symptoms. J Nerv Ment Dis 2008;196:95-9.

17. Green MF, Horan WP, Lee J. Social cognition in schizophrenia. Nat Rev Neurosci 2015;16:620-31.

18. Sprong M, Schothorst P, Vos E, et al. Theory of mind in schizophrenia. Br J Psychiatry 2007;191:5-13.

19. Bora E, Yucel M, Pantelis C. Theory of mind impairment in schizophrenia: metaanalysis. Schizophr Res 2009;109:1-9.

20. Hirao K, Miyata J, Fujiwara H, et al. Theory of mind and frontal lobe pathology in schizophrenia: a voxel-based morphometry study. Schizophr Res 2008; 105:165-74.

21. Li H, Chan RC, Zhao Q, et al. Facial emotion perception in Chinese patients with schizophrenia and non-psychotic first-degree relatives. Prog Neuropsychopharmacol Biol Psychiatry 2010;34:393-400.

22. Bliksted V, Fagerlund B, Weed E, et al. Social cognition and neurocognitive deficits in first-episode schizophrenia. Schizophr Res 2014;153:9-17.

23. Greig TC, Bryson GJ, Bell MD. Theory of mind performance in schizophrenia: diagnostic, symptom, and neuropsychological correlates. J Nerv Ment Dis 2004;192:12-8. 